\title{
Self-stopping actuating mechanism with high coefficient of efficiency of the forward stroke for linear motion drives
}

\author{
Valentin Morozov and Alexey Zhdanov \\ Vladimir State University named after Alexander and Nikolay Stoletovs, Department of Mechanical Engineering, 600000, Vladimir, Russia
}

\begin{abstract}
In this article is examined the an approach to the design of linear motion mechanisms, which consist of selfstopping planetary transmissions of the type $A_{H 1}^{3}$ and $A_{H 3}^{1}$ with transmission values $i=7 \div 9$ based on the type of selfstopping planetary transmissions and the RSM. That combination allows a reliable self-stopping in backward stroke and a high coefficient of efficiency of the forward stroke (up to 0.95). The dependences of the angles of inclination of the wheel of the self-stopping transmissions from the angle of the original profile were lead. The obtained conditions allow the transmissions work of the type $A_{H 3}^{1}$ and $A_{H 1}^{3}$ in release mode for the back-and-forth stroke.
\end{abstract}

\section{Introduction}

Recently the roller-screw mechanism (RSM) are widely used in linear motion drives $[1-4,9,10]$. These mechanisms have a high coefficient of efficiency in backand-forth stroke and the drives that use RSM to hold the load must have different automatic stopping mechanisms, which significantly increases the weight and the dimensions of the drive $[6,11,12]$. The application in such drives of self-stopping sliding screw-nut transmissions and worm gears with a forward stroke efficiency of $<0.5$ dramatically reduces the efficiency of the drive $[5,6]$.

Therefore, the search for new design solutions of actuating mechanism to drives with output linear motion is an actual task.

Self-stopping toothed planetary transmissions are promising for use in actuating mechanisms of such drives [8].

The carried out study of cylindrical transmission showed that, in order to achieve the self-stopping in them, it is necessary to use a self-stopping gear with a significant misalignment. [7, 8] As in the spur gears the possible misalignment of the profiles working areas are very limited because of the risk of tapering or undercutting the teeth, if it is necessary, they are replaced by helical or double helical gears, for which is possible a considerably bigger misalignment. In the helical transmissions the misalignment magnitude of the working profiles from the origin is mainly determined by the difference between the angles of inclination of the teeth profiles, which are part of the engagement [7, 8]. Therefore, the task of ensuring the self-stopping of the planetary transmission in a given state determines the necessary angles of inclination of the teeth in recession engagements. At the same time, the normal profiles angles are selected from the same consideration as in conventional transmissions.

\section{General conditions}

The kinematic diagram of the planetary transmission $A_{H 1}^{3}$ with a one-crowned planetary pinion 2 having a external engagement with the central wheel 1 is shown in Fig. 1a, diagram of forces in the engagements of this transmission is shown in Fig. 2. The forces, acting on the cage $\mathrm{H}$ and the cage itself are not shown in Fig. 2.

We will write the links equilibrium equations in steady flow in the traction mode of the forward stroke when the wheel 1 is the driving one and the cage $\mathrm{H}$ is the driven one:

central wheel 1:

$$
M_{1}=r_{b 1}\left(F_{n t 12}+F_{T 12} \operatorname{tg} \alpha_{t y 12}\right)
$$

planetary pinion 2 :

$$
r_{b 2}\left(F_{n t 21}+F_{T 21} \operatorname{tg} \alpha_{t y 21}\right)=r_{b 2}\left(F_{n t 23}+F_{T 23} \operatorname{tg} \alpha_{t y 23}\right) ;
$$

cage $\mathrm{H}$ :

$$
\begin{aligned}
M_{H}= & F_{T 21}\left(r_{b 1} \operatorname{tg} \alpha_{t y 12}+r_{b 2} \operatorname{tg} \alpha_{t y 21}\right)+F_{n t 21}\left(r_{b 1}+r_{b 2}\right)+ \\
& +F_{n t 23}\left(r_{b 3}-r_{b 2}\right)+F_{T 23}\left(r_{b 3} \operatorname{tg} \alpha_{t y 32}-r_{b 2} \operatorname{tg} \alpha_{t y 23}\right)
\end{aligned}
$$




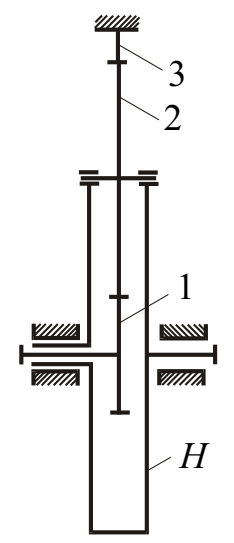

a)

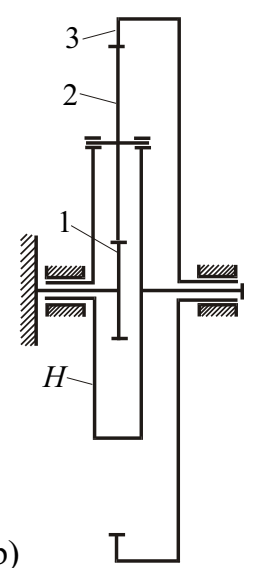

b)

Figure 1. Planetary transmission with one-crowned planetary pinions a transmission $A_{H 1}^{3}, \mathrm{~b}$ transmission $A_{H 3}^{1}$.

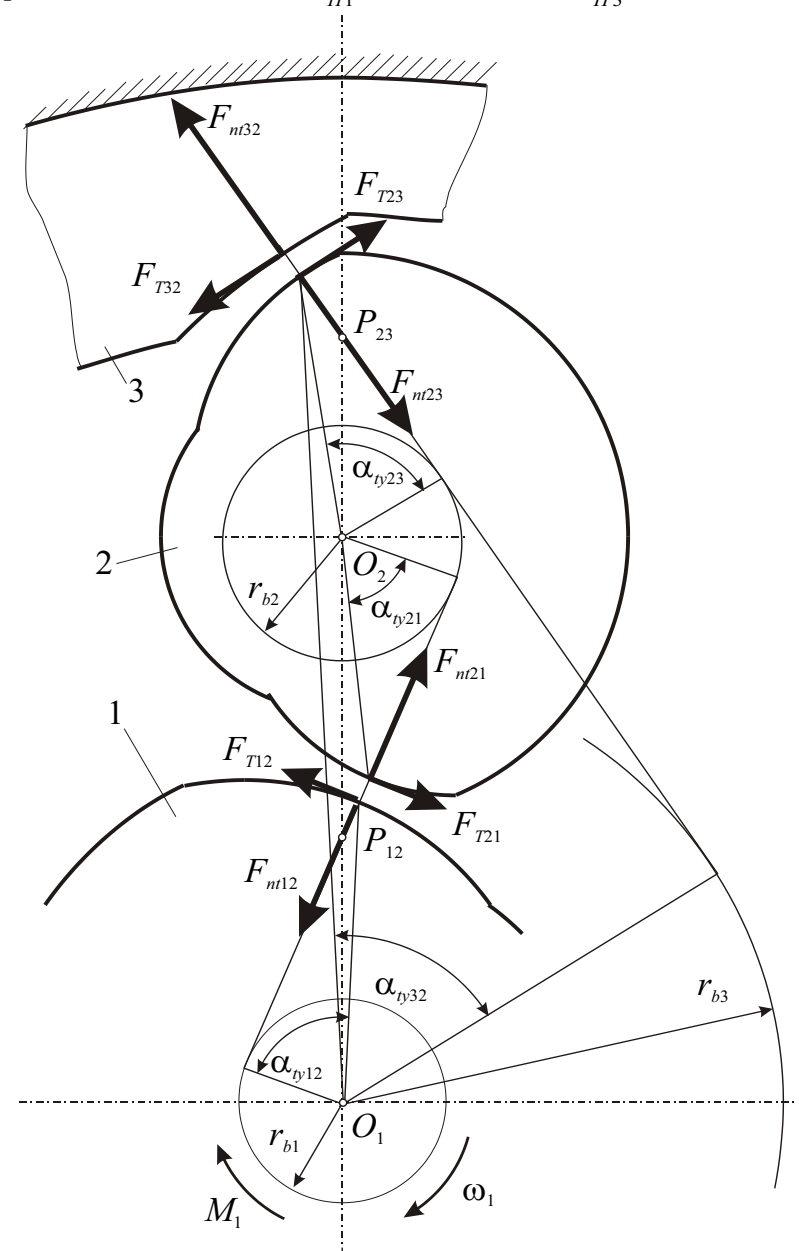

Figure 2. Force diagram of the planetary transmission $A_{H 1}^{3}$.

We will convert the equation (1) ... (3) to:

$$
\begin{aligned}
& M_{1}=F_{n t 12} r_{b 1}\left(1+k_{12}\right) ; \\
& F_{n t 21}\left(1+k_{21}\right)=F_{n t 23}\left(1+k_{23}\right) ;
\end{aligned}
$$

$$
\begin{aligned}
& M_{H}=F_{n t 21}\left[r_{b 1}\left(1+k_{12}\right)+r_{b 2}\left(1+k_{21}\right)\right] \\
& +F_{n t 23}\left[r_{b 3}\left(1+k_{32}\right)-r_{b 2}\left(1+k_{23}\right)\right]
\end{aligned}
$$

where

$$
k_{i j}=\frac{F_{T i j} \operatorname{tg} \alpha_{t i j}}{F_{n t i j}} ; \quad i, j=1,2,3 .
$$

If the friction force is proportional to the normal reactions, then the coefficients $k_{i j}$ are:

$$
k_{i j}=\frac{f_{i j} \operatorname{tg} \alpha_{t i j}}{\cos \beta_{b}} ; \quad i, j=1,2,3 .
$$

Solving the system of equations (4) ... (5), we will find the relation of the input and output moments:

$$
\frac{M_{H}}{M_{1}}=1+\frac{r_{b 3}\left(1+k_{21}\right)\left(1+k_{32}\right)}{r_{b 1}\left(1+k_{12}\right)\left(1+k_{23}\right)}=1+\frac{r_{b 3}}{r_{b 1}} \eta_{12} \eta_{23} .
$$

Multiplying by the transmission ratio, and considering that the main radii are proportional to the number of teeth, we get the known formula [8] for the coefficient of efficiency of forward stroke:

$$
\eta_{1 H}=\frac{1+\frac{z_{3}}{z_{1}} \eta_{23} \eta_{12}}{1+z_{3} / z_{1}} .
$$

From the equations (4) ... (5) and (10) results that the traction mode of the transmission forward stroke $A_{H 1}^{3}$ is possible under simultaneous implementation of the conditions:

$$
-\frac{z_{1}}{z_{3}}<\eta_{23} \eta_{12}<1 ; k_{12}>-1 ; k_{21}>-1 ; k_{23}>-1 .
$$

The realization of the conditions (11) substantially does not impose any further constraints during the design of the transmission, since at the real values of the coefficient of friction in the engagements these conditions won't be completed just for the wheels with very large angles of inclination of the teeth (over $80^{\circ}$ ).

The equations of the links equilibrium in traction mode of the backward stroke differs from the equations (1) ... (3), by the friction forces opposite signs, when $\mathrm{H}$ becomes the leading cage:

central wheel 1:

$$
M_{1}=r_{b 1}\left(F_{n t 12}-F_{T 12} \operatorname{tg} \alpha_{t y 12}\right) ;
$$

planetary pinion 2 :

$$
r_{b 2}\left(F_{n t 21}-F_{T 21} \operatorname{tg} \alpha_{t y 21}\right)=r_{b 2}\left(F_{n t 23}-F_{T 23} \operatorname{tg} \alpha_{t y 23}\right) ;
$$

cage $H$ : 


$$
\begin{aligned}
M_{H}= & F_{n t 21}\left(r_{b 1}+r_{b 2}\right)-F_{T 21}\left(r_{b 1} \operatorname{tg} \alpha_{t y 12}+r_{b 2} \operatorname{tg} \alpha_{t y 21}\right)+ \\
& +F_{n t 23}\left(r_{b 3}-r_{b 2}\right)-F_{T 23}\left(r_{b 3} \operatorname{tg} \alpha_{t y 32}-r_{b 2} \operatorname{tg} \alpha_{t y 23}\right)
\end{aligned}
$$

Introducing the indications according to the formula (7), we convert the equation (12) ... (14) to:

$$
\begin{gathered}
M_{1}=F_{n t 12} r_{b 1}\left(1-k_{12}\right) ; \\
F_{n t 21}\left(1-k_{21}\right)=F_{n t 23}\left(1-k_{23}\right) ; \\
M_{H}=F_{n t 21}\left[r_{b 1}\left(1-k_{12}\right)+r_{b 2}\left(1-k_{21}\right)\right]+ \\
+F_{n t 23}\left[r_{b 3}\left(1-k_{32}\right)-r_{b 2}\left(1-k_{23}\right)\right]
\end{gathered}
$$

Solving the system of equations (15) ... (17), we will find the relation of the input and output moments:

$$
\frac{M_{H}}{M_{1}}=1+\frac{r_{b 3}\left(1-\tau_{21}\right)\left(1-\tau_{32}\right)}{r_{b 1}\left(1-\tau_{12}\right)\left(1-\tau_{23}\right)}=1+\frac{r_{b 3}}{r_{b 1}} \eta_{32} \eta_{21}
$$

Multiplying by the transmission ratio, and considering that the main radii are proportional to the number of teeth, we get the formula for the coefficient of efficiency of backward stroke $\eta_{H 1}$ :

$$
\eta_{H 1}=\frac{1+\frac{z_{3}}{z_{1}}}{1+\frac{z_{3}}{z_{1} \eta_{21} \eta_{32}}}
$$

It should be noted that in the formula (19) the coefficients of efficiency $\eta_{21}$ and $\eta_{32}$ of backward stroke of every link are included, but not the coefficients of the forward stroke. Since the coefficient of efficiency of the backward stroke can be significantly different from the efficiency of the forward stroke, especially in the recession engagements, this circumstance is very essential.

From the equations (15) ... (17) and (19) it follows that during the realization of the wheels $1-2$ to the recession engagement the conditions of the self-stopping transmission while maintaining the workability in release mode are:

$$
-\frac{z_{3}}{z_{1} \eta_{32}}<\eta_{21}<0
$$

From the inequality (20) the parameters $k_{12}$ and $k_{21}$ are expressed, taking into account the friction on the wheels 1 and 2:

$$
\eta_{21}=\frac{1-k_{12}}{1-k_{21}}>-\frac{z_{3}}{z_{1} \eta_{32}}
$$

The parameters $k_{12}$ и $k_{21}$ can be expressed through the generalized coefficient of friction $f_{12}^{0}$ and $f_{21}^{0}$ in the engagement of the wheels 1 and 2, and the normal angles $\alpha_{n y 1}$ и $\alpha_{n y 2}$ of the profiles of the wheels 1 and 2:

$$
k_{i j}=\frac{f_{i j}^{0} \operatorname{tg} \alpha_{n y i}}{\cos \beta_{y i} \cos \beta_{b}} ; i, j=1,2 .
$$

Analysis of (21) shows that it limits the maximum permissible value of the parameter $k_{12}$ :

$$
k_{12 \max }<\frac{z_{3}}{z_{1} \eta_{32}}\left(1-k_{21 \max }\right)
$$

Thus the value $\beta_{y 1}$ must satisfy the condition:

$$
\sec \beta_{y 1}<\frac{\cos \beta_{b}}{f_{12 \max }^{0} \operatorname{tg} \alpha_{n y 1}} \frac{z_{3}}{z_{1} \eta_{32}}\left(1-k_{21 \text { max }}\right)
$$

The equations of equilibrium of the links in the steady-state mode of release are different from (15) ... (17) only by the sign of the input torque M1. Their solution gives the following relation between the output and the input moments:

$$
\frac{M_{H}}{M_{1}}=\frac{r_{b 3}\left(1-k_{21}\right)\left(1-k_{32}\right)}{r_{b 1}\left(1-k_{12}\right)\left(1-k_{23}\right)}-1=-\frac{z_{3}}{z_{1} \eta_{32} \eta_{21}}-1
$$

In this case, when it is necessary to remove the release mode and the mechanism is designed to work only in traction mode of forward stroke, the parameters of the stopping profiles must satisfy the following conditions:

$$
\begin{aligned}
& \cos \beta_{y 1} \leq \operatorname{tg} \alpha_{n y 1} \sqrt{\sqrt{\frac{1}{4}+\left(\frac{f_{12 \min }^{0}}{\sin \alpha_{n y 1}}\right)^{2}}-\frac{1}{2}} \\
& \cos \beta_{y 2} \leq \operatorname{tg} \alpha_{n y 2} \sqrt{\sqrt{\frac{1}{4}+\left(\frac{f_{12 \max }^{0}}{\sin \alpha_{n y 2}}\right)^{2}}-\frac{1}{2}}
\end{aligned}
$$

At the same time the recession condition of the engagements should be saved:

$$
\beta_{y 1}>\beta_{y 2}>\beta_{y 3} .
$$

Kinematic diagram of the planetary transmission $A_{H 3}^{1}$ with a one-crowned planetary pinion 2 having a external engagement with the central wheel 3 is shown in Fig. $1 b$. The coefficient of efficiency of backward stroke $\eta_{H^{3}}$ is:

$$
\eta_{H 3}=\frac{1+\frac{z_{1}}{z_{3}}}{1+\frac{z_{1}}{z_{3} \eta_{12} \eta_{23}}} .
$$

where $\eta_{12} и \eta_{23}$ are the coefficients of efficiency of backward stroke of the engagements of the wheels 2-1 and 3-2 when the cage is stopped. 
During the realization of the wheels 3-2 to the recession engagements, the self-stopping conditions of the transmission that ensure its operation in release mode are:

$$
\begin{gathered}
\cos \beta_{y 2}>\operatorname{tg} \alpha_{n y 2} \sqrt{\sqrt{\frac{1}{4}+\left(\frac{f_{23 \max }^{0}}{\sin \alpha_{n y 2}}\right)^{2}}-\frac{1}{2}} \\
\cos \beta_{y 3} \leq \operatorname{tg} \alpha_{n y 3} \sqrt{\sqrt{\frac{1}{4}+\left(\frac{f_{23 \min }^{0}}{\sin \alpha_{n y 3}}\right)^{2}}-\frac{1}{2}} \\
\sec \beta_{y 3}<\frac{\cos \beta_{b}}{f_{23 \max }^{0} \operatorname{tg} \alpha_{n y 3} \frac{z_{1}}{z_{3} \eta_{23}}\left(1-\max k_{23}\right) .} \\
\beta_{y 3}>\beta_{y 2}>\beta_{y 1}
\end{gathered}
$$

The conditions that ensure the self-stopping transmission operation $A_{H 3}^{1}$ only in traction mode of the forward stroke, similarly to the transmission $A_{H 1}^{3}$ are as follows:

$$
\begin{aligned}
& \cos \beta_{y 3} \leq \operatorname{tg} \alpha_{n y 3} \sqrt{\sqrt{\frac{1}{4}+\left(\frac{f_{23 \min }^{0}}{\sin \alpha_{n y 3}}\right)^{2}}-\frac{1}{2}} \\
& \cos \beta_{y 2} \leq \operatorname{tg} \alpha_{n y 2} \sqrt{\sqrt{\frac{1}{4}+\left(\frac{f_{23 \min }^{0}}{\sin \alpha_{n y 2}}\right)^{2}}-\frac{1}{2}}
\end{aligned}
$$
(33).

\section{Analysis of the research results}

The carried out analysis shows that the transmission $A_{H 1}^{3}$ can be self-stopping during the backward stroke at the first or second variant maintaining high coefficient of efficiency values of the forward stroke. Fig. 3a shows the graphic of the wheels angles of inclination of the selfstopping transmission from the original profile, constructed according to the formula (24). The existence area of the first variant of self-stopping is the strip between the graphs $\beta_{y 1 \text { min }}$ and $\beta_{y 1 \max }$ (two top of the graphic) for the angle of inclination of the tooth of the wheel 1 and the region below the graph $\beta_{y 2 \max }$ for the angle of inclination of the planetary pinion 2 . The existence area of the first variant of self-stopping is the region above the graph $\beta_{y 1 \text { min }}$ for the angle of inclination of the tooth of the wheell and the region above the graph $\beta_{y 2 \max }$ for the angle of inclination of the planetary pinion 2 .

The kind of the existence regions of the first and second variants of self-stopping in the transmission $A_{H 3}^{1}$ is similar to that shown in Fig. 3a.

a)

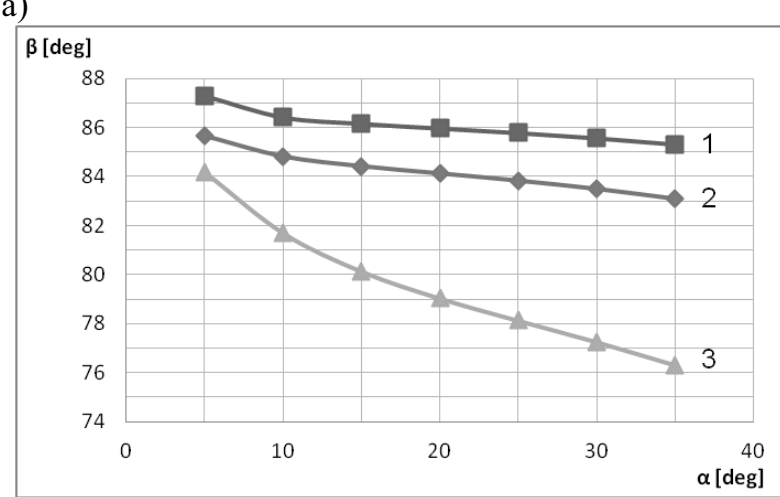

b)

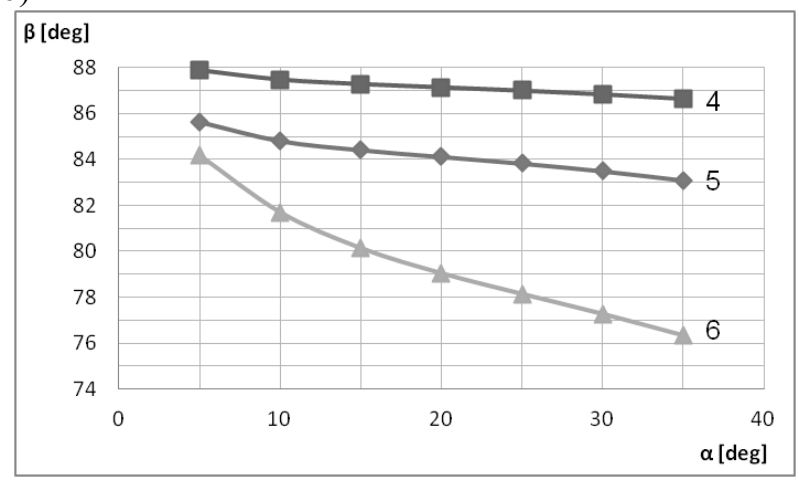

Figure 3. Self-stopping region of the planetary transmissions:

$$
\text { a - transmission } A_{H 1}^{3} ; \mathrm{b}-\operatorname{transmission} A_{H 3}^{1} \text {. }
$$

$\beta$ - angle of inclination of the wheel tooth; $\alpha-$ normal angle of the profile; $1-\beta_{y 1 \text { max }} ; 2-\beta_{y 1 \text { min }} ; 3-$ $\beta_{y 2 \max } ; 4-\beta_{y 3 \max } ; 5-\beta_{y 3 \text { min }} ; 6-\beta_{y 4 \max }$

The application of self-stopping planetary transmissions $A_{H 1}^{3}$ and $A_{H 3}^{1}$ with gear ratios $i=7-9$ and a coefficient of efficiency of forward stroke until 0.98 in conjunction with RSM in the actuating mechanism allows to obtain effective linear motion drives with self-stopping and a coefficient of efficiency of forward stroke until 0.95 .

\section{References}

1. SKF Group, SKF Roller screws: catalogue, (2014)

2. 2. M.H. Jones, S.A. Velinsky, J. Mech. Design, Kinematics of roller migration in the planetary roller screw mechanism, 134, №6 (2012).

3. 3. S.A Velinsky, B. Chu, T.A. Lasky, J. Mech. Design, Kinematics and efficiency analysis of the planetary roller screw mechanism, 131, №1 (2009).

4. 4. V.V. Morozov, A.V. Zhdanov, ICAMEE, pp. 875$881(2015)$ 
5. V.V. Morozov, V.I. Panyukhin, V.V. Panyukhin, V1SU press, Mechanical transmissions: Coefficient of efficiency and self-stopping: Monograph, (2002)

6. V.V. Morozov, VISU press, Roller-screw mechanisms. Kinematic characteristics: Monograph, (2005)

7. V.V. Morozov, A.V. Zhdanov, Rus. Eng. Res., 35, №7 pp. 477-480 (2015)

8. V.I. Panyukhin, Nat. Sci. and Tech. Soc. Press, Selfstopping mechanisms (1981)

9. V.V. Kozyirev, VISU press, Design theory and methods of design and study of planetary gear-nut threaded rollers and mechatronic units based on them: Monograph (2011)

10. P.A Sokolov, O.A Ryakhovskii, D.S. Blinov, A. Laptev, Bulletin Mech. Eng., 1, pp. 3-14 (2005)

11. S. Ma, G. Liu , J. Zhou, R. Tong, Nanjing Un. Sci. And Tech., Optimal design and contact analysis for Planetary Roller Screw (2009)

12. S. Ma, G. Liu, J. Zhou, R. Tong et al., Math. Prob. Eng. New Study on the Parameter Relationship of Planetary Roller Screws, (2012) 